\title{
How can bacteria benefit plants?
}

\author{
Siva Linga Sasanka Velivelli
}

School of Biological, Earth \& Environmental Sciences, UCC

\section{Introduction}

Bacteria are microscopic, single-celled organisms found almost everywhere on earth in vast numbers. They are extremely diverse and play a major role in nature, contributing to plant growth and health. Agriculture provides a major share of the national income in many developing countries. However, diseases cause significant yield and economic losses in many important agricultural crops. Farmers have adopted a strategy to increase crop yields by applying large quantities of chemical fertilizers and pesticides. The use of chemical-based fertilizers offers some protection against plant pathogens and provides immediate relief, but cannot provide a long-term sustainable solution.

The excessive use of chemical-based fertilizers also causes severe environmental problems. Many countries have banned the use of certain hazardous chemicals, including some pesticides that are used to control plant diseases. For example, methyl bromide, used in the control of pests, has been banned internationally because of its adverse effects on human health and the environment. It is very difficult for farmers to produce a good quality and a high yield in economically important agricultural crops with limited control measures. Due to current public concerns about the adverse effects of agrochemicals, new technologies that can improve the economic viability of agricultural systems, with little reliance on the use of chemical fertilizers and pesticides, are urgently needed and should be a high priority for agricultural research, both now and in the immediate future.

An ideal agricultural system is sustainable if it maintains and improves human health, benefits producers and consumers, produces enough food for an increasing world population, while maintaining or enhancing the environmental quality and conserving natural resources such as soil and water, both now and in the immediate future.

Biological control appears to be a potential alternative to chemical fertilizers and pesticides and offers an environmentally friendly approach to the management of plant diseases. Biological control of plant disease is defined as "the involvement of the use of beneficial micro-organisms, such as bacteria to attack, and control the plant pathogens". Plant roots support a wide range of micro-organisms that can have either a deleterious or beneficial impact on plant health and growth. The use of beneficial micro-organisms to control or reduce plant diseases are known as plant-growth-promoting rhizobacteria (PGPRs) ('rhizo' means 'root'). PGPRs have gained worldwide importance over the last 
few years and have attracted much attention in their role in reducing plant diseases, thus playing an important role in crop production. The use of PGPRs to promote plant growth and to control plant diseases continues to be an area of rapidly expanding research.

Scientists have been interested in the use of potential and efficient PGPRs in a way that can enhance soil quality and crop productivity. PGPRs have the capacity to promote plant growth directly or indirectly and play an important role in sustainable agricultural production. There are various mechanisms by which PGPRs affect plant growth directly or indirectly. The direct promotion of plant growth by PGPRs can be through a number of methods.

1. Some plants cannot use atmospheric nitrogen for their growth. PGPRs may fix atmospheric nitrogen into ammonia and supply it to plants for better growth.

2. They also synthesize phytohormones such as indole acetic acid (IAA), gibberellic acid (GA) and cytokinin, which can act to improve the different stages of plant growth.

3. Phosphorus, which is an essential nutrient, can often be a limiting factor for plant growth; however, some PGPRs solubilize insoluble phosphate into soluble forms and make them more accessible for plant growth under poor phosphorus availability.

4. Ethylene is important for plant growth and development and it is required by many plant species for seed germination. High levels of ethylene can lead to inhibition of root elongation. Some PGPRs synthesize an enzyme 1, which can lower plant ethylene levels, thereby increasing root length and growth.

5. Iron deficiency is a limiting factor of plant growth. Some plant-growth-promoting rhizobacteria also play an important role in the production of siderophores (iron carrier molecules, which solubilize and transport iron) that enhance iron availability to plants.

The indirect promotion of plant growth occurs through the suppression of plant pathogens and involves a variety of mechanisms including the production of antagonistic substances such as antibiotics, fungal cell wall lysing enzymes ('lysis' means 'breakdown' or 'to separate'), or hydrogen cyanide, which suppress the growth of fungal pathogens and the production of siderophores that chelate (solubilize or capture) iron, making it unavailable to pathogens. PGPRs with antifungal properties have been identified as a biological control alternative to pesticides for disease suppression.

\section{My methodology}

My research project aims to explore the existing large biodiversity of soil micro-organisms of Andean countries to improve the sustainability, food security, environmental protection and productivity of Andean cropping systems, benefiting rural farming households. 
Commercial rhizobacterial strains were used as a model to develop the system at the laboratory level for the early detection and selection of potential PGPRs which have potential as biocontrol agents in crop production.

A laboratory study was conducted to characterize the beneficial properties of commercial two rhizobacterial (PGPRs) strains of Bacillus subtilis and Pseudomonas fluorescens for their multiple plant growth promoting activities (Fig. 1). The two commercial bacterial strains were tested for their ability to inhibit plant diseases using plate tests. The effects of the PGPR isolates on plant growth promotion and suppression of potato disease in potato microplants were also examined. These commercial rhizobacteria were also tested for various activities which can promote plant growth, including the production of plant growth hormones.

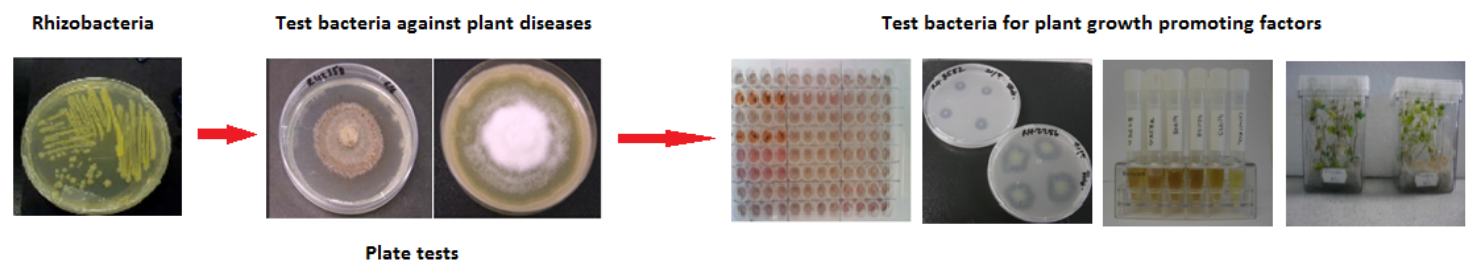

Figure 1: Screening of commercial rhizobacterial (PGPR) strains for their multiple plant growth promoting activities

\section{What did I find?}

This research examines the effect of commercial rhizobacterial (PGPR) strains for their ability to inhibit plant diseases using plate tests and also plant growth promotion and suppression of potato disease in potato microplants. These commercial rhizobacteria influenced plant growth and plant health positively and the inhibition of plant disease could be due to the production of various chemical compounds. They also act as plant growth promoters due to their ability to produce various plant growth hormones. The two commercial rhizobacterial (PGPR) strains inhibited plant disease by $21.32 \%$ and $19.28 \%$. These two rhizobacteria also increased plant growth by $20 \%$ and $12 \%$ and the disease suppression activity was also evaluated in terms of percentage under disease pressure by $15 \%$ and $12 \%$ [Table.1].

\section{Conclusion}

These two commercial rhizobacterial strains inhibit plant disease and also have significant effects on plant growth and disease suppression in laboratory tests. Rhizobacteria that 
Table 1: Plant growth promoting attributes of the commercial PGPR Strains

\begin{tabular}{|c|c|c|c|}
\hline Strain & $\begin{array}{c}\text { Effect on plant } \\
\text { disease \% }\end{array}$ & $\begin{array}{c}\text { Plant growth } \\
\text { promotion \% }\end{array}$ & $\begin{array}{c}\text { Plant disease } \\
\text { suppression \% }\end{array}$ \\
\hline $\begin{array}{c}\text { Bacillus subtilis FZB } \\
\mathbf{2 4}\end{array}$ & 21 & 20 & 15 \\
\hline $\begin{array}{c}\text { Pseudomonas } \\
\text { fluorescens } \text { CHAO }\end{array}$ & 19 & 12 & 12 \\
\hline
\end{tabular}

control plant diseases have gained worldwide importance and are potential tools for sustainable agriculture. Promotion of plant growth by rhizobacteria offers an attractive way to reduce or replace chemical fertilizers and pesticides and also decrease adverse environmental effects. These results indicate the beneficial properties of the rhizobacteria which could be used as a tool for selecting new/potential PGPRs.

Siva Linga Sasanka.Velivelli is a PhD student in the School of Biological, Earth and Environmental Sciences, under the supervision of Dr. Barbara Doyle Prestwich. The author would like to take this opportunity to thank the senior postdoctoral researcher in the lab Dr. Eileen $\mathrm{O}^{\prime}$ Herlihy. The author would also like to acknowledge contributions to this research from Dr. Bogumila Janczura and funding from European Community's Seventh Framework Programme FP7/2007-2013 (Grant No: 227522, 01/02/2009-31/01/2014). The author would also like to thank Ms. Eileen Crowley for proof-reading. More information can be found on VALORAM website: http://valoram.ucc.ie 